\title{
Affine Structure from Translational Motion in Image Sequences
}

\author{
Pär Hammarstedt ${ }^{1}$, Fredrik Kahl ${ }^{2}$, and Anders Heyden ${ }^{1}$ \\ 1 School of Technology and Society \\ Malmo University, Sweden \\ par.hammarstedt@ts.mah.se \\ 2 Centre for Mathematical Sciences, Lund University, Sweden
}

\begin{abstract}
In this paper a method for obtaining affine structure from an image sequence taken by a translating camera with constant intrinsic parameters is presented. A general geometric constraint, expressed using the camera matrices, is derived and this constraint is used in a least squares solution of the problem. The first step is to obtain a projective reconstruction, in the form of a sequence of camera matrices (and a sparse set of feature points), and then these constraints are used to upgrade to an affine reconstruction. The proposed algorithm extends previous results of affine structure recovery from two images with a translating camera to the general case of a sequence of images. The proposed method is illustrated in both simulated and real experiments.
\end{abstract}

Keywords: structure and motion estimation, affine camera, translating motion, stratification

\section{Introduction}

One of the main goals in computer vision is to recover the Euclidean $3 \mathrm{D}$ structure of a scene, where the projective to affine reconstruction upgrade is an essential step [1]. Much work has been done in recovering both structure and motion from image sequences where the motion of the camera as well as the structure of the scene are unknown [2-4]. For these algorithms, it is assumed and often required the camera motion is unconstrained (i.e. the camera movement contains both translation and rotation). In many applications of computer vision, such as car crash tests, vehicle navigation and monitoring of industrial assembly lines, the relative motion of the camera is restricted to a translation. This information can be used in the reconstruction process. In [5] a method is presented to obtain an affine reconstruction from one pair of images taken with a translating camera with constant intrinsic parameters. We will now extend these previous results by showing that given a sequence of two or more images from a translating camera with constant intrinsic parameters, a projective reconstruction of the scene can be upgraded to an affine reconstruction. We will also present an algorithm for doing this.

Three-dimensional reconstruction from a purely translating camera has some inherent limitations. In the case of a translating camera where all the intrinsic parameters are varying and unknown, an affine reconstruction is impossible [6,7]. It is also wellknown that the affine to Euclidean upgrade from translational motion is critical in the 
sense that some extra information is needed in order to get a unique Euclidean structure [8]. However, as such motions are frequent in practice, we need to be able to deal with them.

Several authors have exploited the special form of the camera matrices for translational motion and applications to projective reconstruction. In [9] it is shown that the camera matrices have simple forms when assuming translational motion and also when co-planar points can be utilized, e.g. the projectively reduced setting. Also in [10] coplanar structures are used to simplify the reconstruction algorithm. However, neither of these papers utilizes translating cameras and affine reconstruction from constant intrinsic parameters.

The purpose of this paper is to present an algorithm for affine reconstruction from an image sequence taken by a translating camera. The first step is to estimate the projective structure and motion using standard techniques. The obtained sequence of camera matrices (determined up to an unknown projective transformation) is then upgraded to an affine motion, i.e. a sequence of camera matrices determined up to an affine transformation. The constraints needed to perform this upgrade is derived and a least squares solution is adopted. The paper is organized as follows: In Section 2 some notation and background about projective reconstruction are given. The constraints imposed by assuming translational motion are derived in Section 3 and the least squares solution is presented in Section 4. In Section 5 experiments on both synthetic and real data are presented and in Section 6 we will give some conclusions.

\section{Background and Notation}

We will use the standard pin-hole camera model:

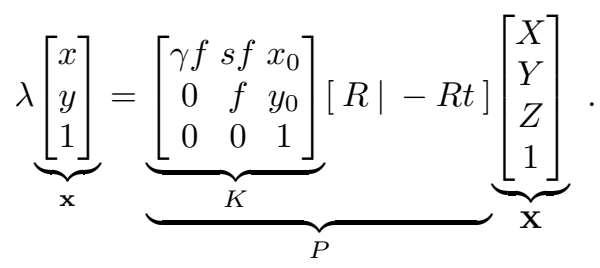

Here, $f$ denotes the focal length, $\gamma$ and $s$ the aspect ratio and the skew and $\left(x_{0}, y_{0}\right)$ the principal point. These are called the intrinsic parameters, and they are contained in the upper-triangular calibration matrix $K$. Furthermore, $R$ and $t$ denote the relation between the camera coordinate system and the object coordinate system, where $R$ is a rotation matrix and $t$ a translation vector, i.e. a Euclidean transformation. The object points in homogeneous coordinates are denoted by $\mathbf{X}$ and the image points in homogeneous coordinates by $\mathbf{x}$.

In a sequence with several images we will use the notation

$$
\lambda_{i} \mathbf{x}_{i}=P_{i} \mathbf{X}, \quad i=1, \ldots m
$$

where $m$ denotes the number of images.

The initial structure and motion is obtained from the following steps: 


\section{Extract and track feature points through the image sequence:}

The standard Harris corner detector, cf. [11] is used together with a correlation-based tracker, similar to the KLT tracker, cf. [12], [13].

\section{Use a robust method to estimate fundamental matrices and trifocal tensors:}

The RANSAC method method is used to estimate the fundamental matrix, $F$, relating corresponding image coordinates in two images, and the trifocal tensor, $T$, relating corresponding image coordinates in three images. In practice, feature points are tracked until the estimated fundamental matrix has a smaller error than an estimated homography according to an information criterion and similarly for the trifocal tensor.

Iteratively, use resection and intersection:

Once a structure is obtained from three key-frames, the camera position for additional frames can be estimated using resection and new feature points can be reconstructed using intersection. Also in this stage a robust method has to be applied to remove false matches and outliers.

\section{Bundle adjustment:}

The maximum likelihood estimate of the projective structure and motion is the solution to the optimization problem

$$
f=\sum_{i, j \in I}\left(\mathbf{x}_{i, j}-g\left(P_{i}, \mathbf{X}_{j}\right)\right)^{2},
$$

where $g\left(P_{i}, \mathbf{X}_{j}\right)$ denotes the re-projected feature points. The minimization of $f$ can be done by iterative methods as described for the bundle adjustment method, [14].

\section{Translational Motion}

In a Euclidean reconstruction, the camera matrices $P_{i}^{E}$ are of the form

$$
P_{i}^{E}=K_{i} R_{i}\left[I \mid-\mathbf{t}_{i}\right] .
$$

Knowing that the camera is a pure translation, one can assume that $R_{i}=I$. Further, assuming constant intrinsic parameters we have

$$
P_{i}^{E}=K\left[I \mid-\mathbf{t}_{i}\right] .
$$

A coordinate transformation, represented by a $4 \times 4 H$, of projective 3D-space is an affine transformation iff it has the following form:

$$
H=\left[\begin{array}{ll}
A & \mathbf{u} \\
0 & 1
\end{array}\right] \text {, }
$$

where $\mathrm{A}$ is a nonsingular $3 \times 3$ matrix. Given the Euclidean motion, $P_{i}^{E}$, and $3 \mathrm{D}$-points $\mathbf{X}_{j}$ which satisfies the camera equations

$$
\lambda_{i j} \mathbf{x}_{i j}=P_{i}^{E} \mathbf{X}_{j}=K\left[I \mid-\mathbf{t}_{i}\right] \mathbf{X}_{j}, \quad \text { for all } i, j,
$$

we can change the coordinate system with the transformation

$$
H=\left[\begin{array}{cc}
K^{-1} & 0 \\
0 & 1
\end{array}\right]
$$


so that the cameras are changed to

$$
P_{i}^{E} H=K\left[I \mid-\mathbf{t}_{i}\right] H=\left[K K^{-1} \mid-K \mathbf{t}_{i}\right]=\left[I \mid \mathbf{b}_{i}\right] .
$$

and the points are transformed by $\mathbf{X}_{j} \rightarrow H^{-1} \mathbf{X}_{j}$. Notice that this is also a valid reconstruction since the projection equations are still satisfied. Therefore, since $K$ is unknown (and constant), it is only possible to reconstruct the scene up an unknown affine transformation and one can without loss of generality assume that an object coordinate system has been chosen such

$$
P_{1}^{A}=[I \mid 0] \text { and } P_{i}^{A}=\left[I \mid \mathbf{b}_{i}\right], \quad i>1 .
$$

Given the projective structure and motion, obtained as described above, in the form of a sequence of camera matrices $\left\{P_{i}^{P}\right\}_{i=1 \ldots n}, P_{i}^{P}=\left[A_{i} \mid \mathbf{p}_{i}\right]$, known up to an unknown scale factor and reconstructed feature points $\mathbf{X}_{j}$, our task is now to find a projective transformation, represented by a general non-singular $4 \times 4$ matrix, such that the transformed camera matrices $P_{i}^{P} H$ take the canonical form in (4). Again, without loss of generality we can assume that the projective coordinate system is chosen such that $P_{1}^{P}=[I \mid 0]$. Let

$$
H=\left[\begin{array}{cc}
A & \mathbf{b} \\
\mathbf{v}^{T} & s
\end{array}\right]
$$

where $A$ is a $3 \times 3$ matrix, $\mathbf{b}, \mathbf{v}$ are 3 -vectors and $s$ is a scalar. The first camera matrix gives

$$
P_{1}^{P} H=P_{1}^{A} \quad \Leftrightarrow \quad[I \mid 0]\left[\begin{array}{cc}
A & \mathbf{b} \\
\mathbf{v}^{T} & s
\end{array}\right]=[I \mid 0] \quad \Leftrightarrow \quad[A \mid \mathbf{b}]=[I \mid 0],
$$

which implies that $A=I$ and $\mathbf{b}=0$, giving

$$
H=\left[\begin{array}{cc}
I & 0 \\
\mathbf{v}^{T} & s
\end{array}\right]
$$

For the other cameras $P_{i}^{P}=\left[A_{i} \mid t_{i}\right]$ we get

$$
P_{i}^{P} H \sim P_{i}^{A} \quad \Leftrightarrow \quad\left[A_{i} \mid \mathbf{p}_{i}\right]\left[\begin{array}{cc}
I & 0 \\
\mathbf{v}^{T} & s
\end{array}\right] \sim\left[I \mid \mathbf{b}_{i}\right] \quad \Leftrightarrow \quad\left[A_{i}+\mathbf{p}_{i} \mathbf{v}^{T} \mid s \mathbf{p}_{i}\right] \sim\left[I \mid \mathbf{b}_{i}\right],
$$

where $\sim$ means equality up to scale, implying

$$
A_{i}+\mathbf{p}_{i} \mathbf{v}^{T} \sim I \quad \text { and } \quad \mathbf{b}_{i} \sim \mathbf{p}_{i}
$$

Thus we obtain a linear constraint on the vector $\mathbf{v}$ in the transformation matrix $H$, of the form $A_{i}+\mathbf{p}_{i} \mathbf{v}^{T} \sim I$. If we can calculate $\mathbf{v}$ we also have $H$ and we can upgrade the projective reconstruction to affine by multiplying $P_{i}^{P}$ with

$$
H_{a}=\left[\begin{array}{cc}
I & 0 \\
\mathbf{v}^{T} & 1
\end{array}\right]
$$

In summary, we have the following algorithm for affine reconstruction from translational motion: 
1. Projective reconstruction $P_{i}^{P}=\left[A_{i} \mid \mathbf{p}_{i}\right]$ using traditional techniques.

2. Estimate $\mathbf{v}$ using $A_{i}+\mathbf{p}_{i} \mathbf{v}^{T} \sim I$.

3. Form $H_{a}=\left[\begin{array}{cc}I & 0 \\ \mathbf{v}^{T} & 1\end{array}\right]$

4. Upgrade the reconstruction to affine, $P_{i}^{A}=P_{i}^{P} H_{a}=\left[I \mid \mathbf{b}_{i}\right]$.

In the next section we show how $\mathbf{v}$ can be estimated using a least squares estimate of $\mathbf{v}$.

\section{A Least Squares Solution}

The second step in the algorithm presented above requires us to solve for $\mathbf{v}$ in the overdetermined linear system of equations $A_{i}+\mathbf{p}_{i} \mathbf{v}^{T} \sim I$. We can rewrite this as

$$
A_{i}+\mathbf{p}_{i} \mathbf{v}^{T}=\lambda_{i} I
$$

where $\lambda_{i}$ denote unknown scale factors. We now have $n$ equations of the form

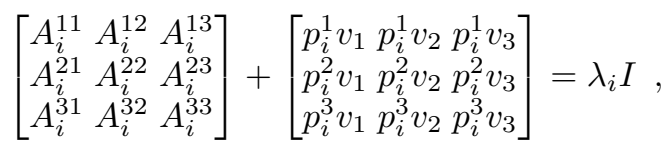

where $A_{i}^{j k}$ denote element $(j, k)$ of $A_{i}$ and similarly for $p_{i}^{k}$. Eliminating $\lambda_{i}$ from (5) we obtain the following equations

$$
\begin{cases}A_{i}^{j k}+p_{i}^{j} v_{k}=A_{i}^{(j+1)(k+1)}+p_{i}^{j+1} v_{k+1}, & j=k, \quad j \neq 3, \\ A_{i}^{j k}+p_{i}^{j} v_{k}=0, & j \neq k,\end{cases}
$$

containing 8 linearly independent equations. Rewriting these into one matrix equation we get

$$
M\left[\begin{array}{c}
v_{1} \\
v_{2} \\
v_{3} \\
1
\end{array}\right]=0 \text { where } M=\left[\begin{array}{c}
M_{2} \\
M_{3} \\
\vdots \\
M_{i} \\
\vdots \\
M_{n}
\end{array}\right] \quad \text { and } M_{i}=\left[\begin{array}{cccc}
p_{i}^{1} & -p_{i}^{2} & 0 & A_{i}^{11}-A_{i}^{22} \\
0 & p_{i}^{2} & -p_{i}^{3} & A_{i}^{22}-A_{i}^{33} \\
p_{i}^{1} & 0 & -p_{i}^{3} & A_{i}^{11}-A_{i}^{33} \\
0 & p_{i}^{1} & 0 & A_{i}^{12} \\
0 & 0 & p_{i}^{1} & A_{i}^{13} \\
p_{i}^{2} & 0 & 0 & A_{i}^{21} \\
0 & 0 & p_{i}^{2} & A_{i}^{23} \\
p_{i}^{3} & 0 & 0 & A_{i}^{31} \\
0 & p_{i}^{3} & 0 & A_{i}^{32}
\end{array}\right],
$$

where three equations have been used for the first constraint in (6) - even if there exists only two linearly independent constraints - because of symmetry and numerical stability. Note that we do not include $M_{1}$ in the construction of $\mathrm{M}$ since all the coefficients in $M_{1}$ will be zero. Let $\mathbf{x}^{T}=\left(v_{1}, v_{2}, v_{3}, 1\right)$. The least squares solution to the above optimization problem

$$
\min _{\|\mathbf{x}\|=1}\|M \mathbf{x}\|
$$

is given by $\mathbf{x}=$ (the last column in $V$ ), where $V$ is the right unitary matrix in the singular value decomposition of $M$, given by $M=U \Sigma V^{T}$. Since

$$
\mathbf{x}=x_{4}\left[\mathbf{v}^{T} 1\right]^{T},
$$

we can now determine $\mathbf{v}$ in a least squares sense from the $n$ camera matrices. 


\section{Experiments}

To verify the theoretical results, a simulated experiment was performed by generating a sequence of 10 camera matrices $P_{i}^{P}=K\left[I \mid \mathbf{t}_{i}\right] H$, where $K$ are random but constant intrinsic camera parameters, $\mathbf{t}_{i}$ represents a translational motion in a random direction with a randomly varying speed, and $H$ is a random nonsingular projective transformation matrix. The camera matrices $P_{i}^{P}$ now represent a reconstruction of a translating object up to an unknown projective transformation. After normalization to $P_{i}^{P}=\left[A_{i} \mid \mathbf{p}_{i}\right]$ where $P_{1}^{P}=[I \mid 0]$, the presented method was successfully used to obtain an affine reconstruction, resulting in $P_{i}^{A}=\left[I \mid \mathbf{b}_{i}\right]$.

In a second experiment, a sequence of 20 images of a stationary scene were captured using a translating camera. Figure 1 shows two images from the image sequence and the affine reconstruction.
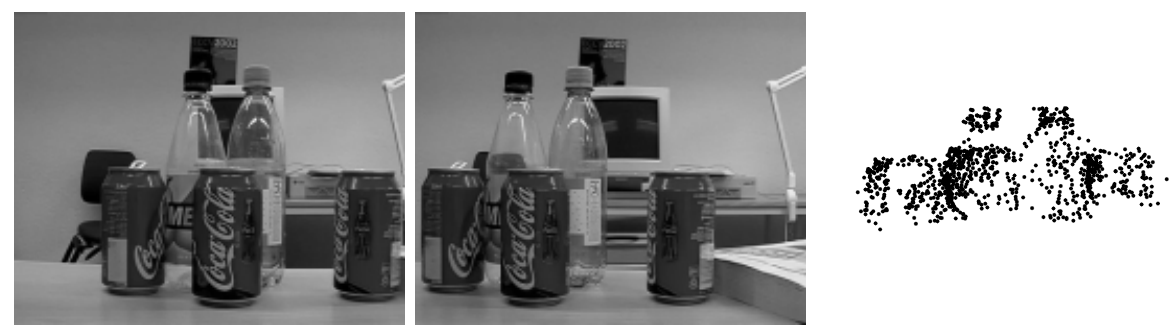

Fig. 1. Two images from an image sequence obtained by a translating camera (left) and some of the reprojected points from the affine reconstruction (right).

A projective reconstruction of the scene were obtained using a standard reconstruction algorithm, and the resulting camera matrices were upgraded from projective to affine using the algorithm derived above. The reprojected 3D points are shown in Figure 1 , and we note that parallelism seems to be preserved, a characteristic of affine transformations. Figure 2 shows a vrml object from a Euclidean reconstruction, which we obtained by assuming reasonable intrinsic parameters for the camera. It was created from the reprojected image points and texture mapped using a Delaunay triangulation of the image points and the texture from one of the images.

A standard measure of the quality of the reconstruction is the root mean square (RMS) of the reprojection errors (which we will denote by $e$ ) an image or in this case in an image sequence. For the projective reconstruction we had $e_{1}=0.2448$ pixels, while after the affine reconstruction the RMS of the reprojection error was $e_{2}=0.3403$ pixels. After applying a bundle adjustment changing only the structure, we had $e_{3}=0.2406$ pixels, showing the stability in the presented algorithm. 

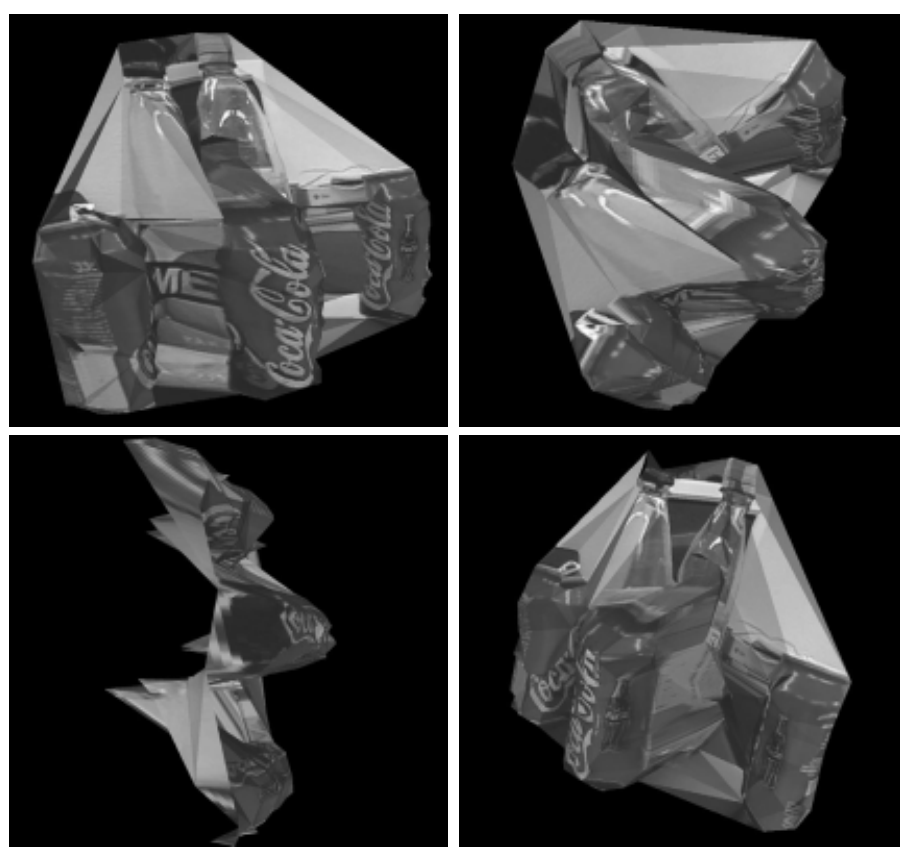

Fig. 2. The reconstructed scene.

\section{Conclusions}

In this paper we have shown that it is possible to make an affine reconstruction from a translating camera. We have presented an algorithm that utilizes all images in a translating image sequence, not only a stereo pair. The algorithm is based on a standard projective reconstruction, followed by a least squares solution based on the constraints evolving from the assumption of a translating camera. The proposed algorithm has been successfully demonstrated on both synthetic and real data. Future research would focus on implementing a bundle adjustment algorithm that gives the optimal affine reconstruction and investigate constraints needed to upgrade to a Euclidean structure.

\section{References}

1. Faugeras, O.: Stratification of three-dimensional projective, affine and metric representations. J. Opt. Soc. America 12 (1995) 465-484

2. Hartley, R., Zisserman, A.: Multiple View Geometry in Computer Vision. Cambridge University Press (2000)

3. Heyden, A., Åström, K.: Flexible calibration: Minimal cases for auto-calibration. In: Int. Conf. Computer Vision, Kerkyra, Greece (1999) 350-355

4. Pollefeys, M., Koch, R., Van Gool, L.: Self-calibration and metric reconstruction in spite of varying and unknown internal camera parameters. In: Int. Conf. Computer Vision, Mumbai, India (1998) 90-95 
5. Moons, T., Gool, L.V., Proesmans, M., Pauwels, E.: Affine reconstruction from perspective image pairs with a relative object - camera translation in between. Application of invariance in Computer Vision (1994)

6. Hu, Z.Y., Wu, F.C.: The impossibility of affine reconstruction from perspective image pairs obtained by a translating camera with varying parameters. In: Proc. Asian Conf. on Computer Vision. (2002)

7. Kahl, F., Triggs, B., Åström, K.: Critical motions for auto-calibration when some intrinsic parameters can vary. Journal of Mathematical Imaging and Vision 13 (2000) 131-146

8. Sturm, P.: Critical motion sequences for monocular self-calibration and uncalibrated Euclidean reconstruction. In: Conf. Computer Vision and Pattern Recognition, San Juan, Puerto Rico (1997) 1100-1105

9. Heyden, A., Åström, K.: Simplifications of multilinear forms for sequences of images. ivc 15 (1997) 749-757

10. Rother, C., Carlsson, S.: Multi view reconstruction and camera recovery using a reference plane. ijcv 49 (2002) 117-141

11. Harris, C., Stephens, M.: A combined corner and edge detector. In: Alvey Vision Conference. (1988) 147-151

12. Lucas, B.D., Kanade, T.: An iterative image registration technique with an application to stereo vision. In: Int. Journal of Computational and Artificial Intelligence. (1981) 674-579

13. Shi, J., Tomasi, C.: Good features to track. In: Proc. Int. Conf. on Computer Vision. (1994) 573-600

14. Slama, C., ed.: Manual of Photogrammetry. 4:th edn. American Society of Photogrammetry, Falls Church, VA (1984) 